\title{
GLOBULAR CLUSTER LUMINOSITY FUNCTIONS
}

\author{
Pierre Demarque \\ Yale University Observatory
}

\begin{abstract}
The use of luminosity functions in the following areas is reviewed: (a) the determination of the helium content and ages of the globular clusters; (b) the testing of stellar structure theory; and (c) the determination of the initial mass function of globular clusters.
\end{abstract}

\section{INTRODUCTION}

Together with color-magnitude (CM) diagrams, the luminosity functions (LF) of globular clusters (GC) have historically been the primary interface between observation and theory in the study of stellar evolution. It was realized early that LF's provide us with the means to test empirically the rate of stellar evolution (Sandage 1957), and to derive the stellar birthrate function (Salpeter 1955; Schwarzschild 1958).

During the next quarter century, however, there were few attempts to compare stellar evolution theory directly with observed LF's, or to use LF's to derive the properties of GC's (see Paczynski's 1984 discussion). The traditional techniques of photographic photometry were too laborious, insufficiently accurate, and subject to too many unavoidable systematic errors. At the same time, the development of photoelectric photometry made more precise calibration of the main sequence turnoff possible, albeit for stars observed one-at-a-time. The main research emphasis then naturally shifted to the construction and interpretation of CM-diagrams, and remarkable progress was made in our understanding of stellar evolution and in the sophistication of our determinations of GC ages (Sandage 1969; Demarque and McClure 1977; Demarque 1980; Sandage 1982; Janes and Demarque 1983; VandenBerg 1983; Green et al.1987).

There were exceptions, however. Some attempts were made during that period to evaluate the GC initial helium content using the subgiant LF. I shall return to this topic in the next section. In a detailed study, Green (1981) constructed LF's for the brighter end of the giant branch of a number of GC's, based on the available data in 
the literature. Green's LF's, which were by necessity coarsely binned, agreed fairly well with the predictions of theory, although a small number of clusters appeared to be deficient in bright giants.

In the last few years, thanks to new computerized techniques which exploit the full power of PDS microdensitometers and of CCD panoramic detectors (Stetson 1979a, 1979b), it has become possible to construct high-precision LF's. As a result, we are now witnessing a veritable revolution in GC LF research, which promises to transform our understanding of a number of key areas in stellar evolution, stellar dynamics, and the evolution of stellar systems.

Most of the interest is currently concentrated in three directions:

(1) Researchers look to LF's to help set more stringent constraints not only on the helium abundance, but also on the metallicities and ages of individual globular clusters. The aim here is (a) to answer the cosmologically significant question of the age of the oldest stars, and (b) to understand better the formation and chemical evolution of the galactic halo.

(2) Accurate GC LF's can teach us about the internal structural changes taking place in the interiors of low mass stars as they evolve. We are now finding fine features on the giant branch LF which are unexplained. There are also unexplained CNO abundance variations, which have been known for some time among GC giants (Kraft 1979; Freeman and Norris 1981), some of which are probably due to rotationally induced internal mixing (Sweigart and Menge1 1979). The resolution of these problems would greatly strengthen our confidence in current models and in the cluster chronology which we derive from them.

(3) The LF for the unevolved main sequence yields the slope of the initial mass function (IMF) in each GC. One hopes thus to gain information on the processes of star formation and on the parameters which control the form of the IMF. An understanding of these parameters, together with reliable evolutionary lifetimes for stars of different chemical compositions and ages, is essential to unravel the history of the Galaxy and nearby systems (Larson 1986; Scalo 1986). The same data are also necessary to interpret the integrated light of distant stellar systems through the construction of population models for these systems (Tinsley 1980; Gunn et al.1981).

\section{THE MAIN SEQUENCE TURNOFF AND SUBGIANT PHASES}

\subsection{Helium.}

The initial aim, in the mid-and late-sixties, was to distinguish between "low" Y, i.e. Y 0.1 , as seemed appropriate if all the helium in the Universe had been synthesized in stars, or "high" Y, 1.e. $Y<0.25$, if helium production had taken place in a primordial fireball (Hoyle and Tayler 1964). When it became clear that the spectroscopy of blue horizontal-branch (HB) stars could not resolve this problem [ $i . e$. the low surface helium abundances observed in the 
atmospheres of these stars are not representative of the initial $\mathrm{Y}$ in GC's (Sargent and Searle 1967)], several attempts were made to derive helium abundances from the slope of the subgiant LF in GC's (Simoda and Kimura 1968; Simoda and Tanikawa 1970; Hartwick 1970; Faulkner 1972).

Figures 1 and 2, taken from the work of Green et al. (1987), illustrate the effect of a change in $Y$ on the GC LF, for a given metallicity. Provided a sufficiently large sample of stars can be measured (about two thousand stars in a three-magnitude interval above the turnoff), an accuracy of 0.05 in $Y$ can be achieved (Cole et al. 1983). Of course, comparison of observation with theory is not only limited by the size of the observed sample. At a higher level of precision, some uncertainties in the theory are also likely to become a factor, such as the effect of molecular opacities, which affect calculated radii, and the rotational state of the interior, which may cause sufficient mixing and structural changes to modify evolutionary rates [e.g. see the work of Endal and Sofia (1981) on the evolution of the rotating Sun].

\subsection{Metallicities.}

The subgiant LF also depends on metallicity, as illustrated in Figures 2 and 3 , where two sets of theoretical LF's with different metallicities, but the same helium content, are shown. Although not a strong test of metallicity by itself, the LF provides a useful consistency check when used in conjunction with a fit in the CM-diagram, both of the turnoff and the HB, and together with spectroscopic data and other population indices such as variable stars [see e.g. the work of VandenBerg (1987) described in these Proceedings].

\subsection{Ages.}

In principle, main sequence and subgiant LF's present major advantages for GC age determinations:

(a) the LF is only weakly affected by the uncertainties in the calculated radii of models for late-type stars (the mixing-length problem in the convective envelope).

(b) the nuclear and opacity data which control the rate of evolution near the main sequence are relatively well known at this point, and the effects of their uncertainties are minimized in LF's. This point is well illustrated by a comparison of LF's constructed over a period of years by a number of independent workers, using different opacity data and physical assumptions. The agreement is close and better than for the turnoff position in the HR-diagram [e.g. the work of Simoda and Iben (1970), Ciardullo and Demarque (1977), VandenBerg (1986), Ratcliff (1986) and Green et al. (1987)].

Figures $1-3$ show the sensitivity of the LF on age, for a given set of composition parameters. We see that the LF depends equally sensitively on age and on chemical composition. Thus together with an independent spectroscopic or photometric metallicity estimate, the LF 
Fig. 1. Theoretical LF's for $Y=0.20$ and $Z=0.0001$, from Green et al (1987). The mass function is the Salpeter function, with $S=2.35$. Ages in Myrs are shown for each LF.

Fig. 2. Same as Fig. 1, but for $\mathrm{Y}=0.30$ and $\mathrm{Z}=0.0001$.

Fig. 3. Same as Fig. 1, but for $Y=0.30$ and $Z=0.004$.

Fig. 4. Bolometric magnitude of the LF peak of a function of cluster age and [ $\mathrm{Fe} / \mathrm{H}]$ for models with convective overshoot (thick lines) and without convective overshoot (thin lines), after King et a1. (1985). In each case $Y=0.25$. The observed position of the LF peak for 47 Tuc and NGC 362 are marked with error bar estimates.

provides an important consistency check on a GC's age. How accurate these LF ages are is unknown at this point, since they are also of course subject to the limitations of present theory and the possible effects of rotation and internal mixing.

So far, for GC age determinations in the Galaxy, CM-diagrams have been used nearly exclusively, simply because it has not been practical until recently to construct LF's down to faint magnitudes. In the context of extra-galactic research, where CM-diagrams are much more difficult to construct, LF's are likely to become a more powerful probe of age than CM-diagrams. We note that LF's have already been used very effectively in studies of the stellar population within the Large Magellanic Cloud (Butcher 1977; Stryker 1984).

\section{GAPS AND PEAKS IN THE LF AS PROBES OF STELLAR STRUCTURE}

Observers have for a long time searched for gaps and peaks in the CM-diagrams of star clusters. Except near the main sequence turnoff where large samples of stars are usually available, this pursuit has been fraught with dangers because of the errors due to traditional photographic photometry and to small number statistics. Bahcall and Yahil (1972) have emphasized this last problem in their reanalysis of the giant branch data for M15 in which Sandage, Katem and Kristian (1968) had suggested the existence of gaps on the giant branch. But as mentioned earlier, it is now possible to do much better than a decade ago for at least some clusters using current techniques, which permit more accurate measurements of a statistically significant number of stars, down to fainter magnitudes ( $\mathrm{Da}$ Costa and Villumsen 1981; King et al 1985).

We will consider here in turn two fine structure features which have been detected on the giant branch LF of some GC's: first a peak, discussed by Thomas (1967) and Iben (1968), which is predicted by standard stellar evolution theory; and then a gap (or dip), the interpretation of which is still unclear.

\subsection{The Thomas peak.}

This peak is due to a temporary slowdown in the rate of luminosity increase (for some chemical compositions, there is even a temporary decrease in luminosity), along the giant branch. This change in evolutionary rate is the result of the passage of the hydrogen-burning shell through a step in the run of the helium abundance created earlier deep in the envelope of the star. The step itself is due to the deepening of the convective envelope into layers in which some hydrogen has previously been converted into helium, during the main sequence phase. It coincides in the star with the point of maximum mass penetration of the convective envelope, which occurs near the base of the giant branch in the HR-diagram.

Figures 1 - 4 show that the Thomas peak luminosity is a function of helium content, metallicity and age. Figure 4, borrowed from the work of King et al (1985), illustrates the interdependence predicted 
by theory between these three variables and how, given any two of them, the third one can be derived from the position of the peak.

A careful comparison of the observed peak position in 47 Tuc with standard theoretical models for the correct composition parameters, shows that the convection zone depth of standard red giant models is too shallow. To reproduce the observations, one must introduce some overmixing at the bottom of the convection zone, of the order of one pressure scale height (King et al.1985). The same conclusion is reached for the NGC362 data of Harris (1982).

\subsection{The gap near the base of the giant branch.}

A gap has been found near the base of the giant branch of all GC's of low metallicity for which we have statistically significant data, i.e. by Cannon and Lee (1981) in NGC6752, by Da Costa and Villumsen (1981) in Omega Centauri, and by Buonanno et a1. (1984) in NGC288. On the other hand, 47 Tuc, the only metal-rich cluster which has been similarly searched shows no such feature in its LF.

This gap must correspond to a temporarily rapid phase of evolution. No such rapid phase is encountered in standard evolutionary tracks. Yahil and van den Horn (1985) have noted that at this point there is a discontinuous transition between two different classes of solutions of the hydrostatic equations of stellar structure. It is not impossible that this transition could give rise in some stars to a phase of rapid internal readjustment which is not adequately described by the numerical models.

By analogy with the Thomas peak, a discontinuity in chemical composition through which the hydrogen burning shell might pass could also cause the observed gap. Armandroff and Demarque (1984) have proposed a plausible, although adhoc, model based on the creation of a discontinuity in He3 abundance due to rapid internal mixing.

Unfortunately, this mechanism, like the previous one, does not explain in an obvious way the apparent dependence of the gap's magnitude on metallicity.

At the writing of this paper, it appears that the solution of the gap problem may be contained in still preliminary calculations of helium diffusion in the envelopes of extreme halo population stars, which predict a rapid dredge-up phase of the diffused helium back to the stellar surface as the convective envelope deepens at the base of giant branch (Deliyannis and Demarque 1987). One of the attractive features of this model is that the metallicity dependence of the gap magnitude is a natural consequence of the different rates of internal helium diffusion in metal-rich and metal-poor dwarfs in this temperature range. In this connection, I should mention that several researchers in our group are now concurrently studying the effects of convective, diffusive and rotationally induced mixing on the evolution of low mass stars. We hope that this work will clarify the factors which determine the luminosity of the Thomas peak, the nature of the gap, as well as the origin of the variable CNO abundances observed among GC stars (Freeman and Norris 1981). 


\section{THE LOWER MAIN SEQUENCE LF AND THE IMF}

Very recently, a veritable information explosion in the area of faint star photometry in crowded fields has occurred, thanks to the advent of sophisticated software such as DAOPHOT. Deep main sequence LF's have been published by Fahlman et al. (1985), McClure et al. (1985, 1986), Buonanno et al, (1986), Lupton and Gunn (1986), Richer and Fahlman (1986), Penny and Dickens (1986), and Smith et al. (1986). In most cases, the exponent of the IMF has been derived, assuming a simple power law. McClure et al. (1986), on the basis of fitted power laws for seven clusters, concluded that the exponents decrease with increasing metallicity. However, the very metal-poor cluster NGC6397, studied earlier by $\mathrm{Da}$ Costa (1982), violates this trend. A meaningful comparison between clusters is complicated by dynamical effects, most particularly mass segregation which affects the star counts. Dynamical models, such as the multi-component King (1966) models studied by Da Costa and Freeman (1976) and Gunn and Griffin (1979), already have shed some light on this problem, but it may be that increasingly sophisticated models will have to be constructed to answer the IMF question conclusively. In particular, we need more detailed estimates of the effects of mass segregation in the outer layers of the cluster, where most of the star counts for LF's are made. Work in this area will not only improve our understanding of cluster dynamics, but will also certainly have far-reaching implications for our understanding of the history of stellar populations in the Galaxy.

Our research on stellar LF's has been supported in part by grants AST83-06143 from the National Science Foundation, and NAGW-778 from the National Aeronautics and Space Administration.

\section{REFERENCES}

Armandroff, T. E. and Demarque, P. 1984 Astron. Astrophys. 139, 305. Bahcall, J. B. and Yahil, A. 1972 Astrophys, J. 177, 647.

Buonanno, R., Corsi, C. E., Fusi Pecci, F., Alcaino, G. and Liller, W. 1984 Astrophys. J. 277, 220.

Buonanno, R., Corsi, C. E., Iannicola, G. and Fusi Pecci, F. 1986 Astron. Astrophys. 159, 189.

Butcher, H. R. 1977 Astrophys. J. 216, 372.

Cannon, R. D. and Lee, S. W. 1981 in IAU Colloquium 68,

Astrophysical Parameters for Globular clusters A. G. D. Philip

and D. S. Hayes, eds., L. Davis Press, Schenectady, p. 501.

Ciardullo, R. B. and Demarque, P. 1977 Trans. Yale Univ. Obs. 33.

Cole, P. W. , Demarque, P. and Green, E. M. 1983 in ESO Workshop on Primordial Helium P. A. Shaver, E. Kunth and K. Kjar, eds., p. 235.

Da Costa, G. S. 1982 Astron. J. 87, 990.

Da Costa, G. S. and Freeman, K. C. 1976 Astrophys. J. 84, 752.

Da Costa, G. S. and Villumsen, J. V. 1981 in IAU Colloquium 68, Astrophysical Parameters for Globular clusters A. G. D. Philip and 
D. S. Hayes, eds, L. Davis Press, Schenectady, p. 527.

Deliyannis, C. and Demarque, P. 1987, to be published.

Demarque, P. 1980 in IAU Symposium 85. Star Clusters J. E. Hesser, ed., Reide1, Dordrecht, p. 385.

Demarque, P. and McClure, R. D. 1977 in The Evolution of Galaxies and Stellar Populations B. M. Tinsley and R. B. Larson, eds., Yale University Observatory, p. 199.

Enda1, A. S. and Sofia, S. 1981 Astrophys. J. 243, 625.

Fahlman, G. G., Richer, H. B. and van den Bergh, D. A. 1985 Astrophys. J. Supp1. 58, 225.

Faulkner, J. 1972 Nature Phys. Sci. 235, 27.

Freeman, K. C. and Norris, J. 1981 Ann. Rev. Astron. Astrophys. $19,319$.

Green, E. M. 1981 Ph.D. dissertation, Univ. of Texas, Austin.

Green, E. M., Demarque, P. and King, C. R. 1987 Revised Yale Isochrones and Luminosity Functions in preparation.

Gunn, J. E. and Griffin, R. F. 1979 Astron. J. 84, 752.

Gunn, J. E., Stryker, L. L. and Tinsley, B. M. 1981 Astrophys. J. 249,48 .

Harris, W. E. 1982 Astrophys. J. Supp1. 50, 573.

Hartwick, F. D. A. 1970 Astrophys. J. 161, 845.

Iben, I. J. 1968 Nature 220, 143.

Janes, K. A. and Demarque, P. 1983 Astrophys. J. 264, 206.

King, C. R., Da Costa, G. S. and Demarque, P. 1985 Astrophys. J. 299,674 .

King, I. R. 1966 Astron. J. 71, 64.

Kraft, R. P. 1979 Ann. Rev. Astron. Astrophys. 17, 309.

Larson, R. B. 1986 Monthly Notices Roy Astron. Soc. 218, 409.

Lupton, R. H. and Gunn, J. E. 1986 Astron. J. 91, 317.

McClure, R. D., Hesser, J. E., Stetson, P. B. and Stryker, L. L. 1985 Pub1. Astron. Soc. Pacific 97, 665.

McClure, R. D., van den Bergh, D. A., Smith, G. H., Fahlman, G. G., Richer, H. B., Hesser, J. E., Harris, W. E., Stetson, P. B. and Bell, R. A. 1986 Astrophys. J. 307, L49.

Paczynski, B. 1984 Astrophys. J. 284, 670.

Penny, A. J. and Dickens, R. J. 1986 Monthly Notices Roy. Astron. Soc. 220,845 .

Ratcliff, S. 1986, preprint.

Richer, H. B. and Fahlman, G. G. 1986 Astrophys. J. 304, 273.

Salpeter, E. E. 1955 Astrophys. J. 121, 161.

Sandage, A. R. 1957 Astrophys. J. 125, 422.

Sandage, A. R. 1969 Astrophys. J. 157, 515.

Sandage, A. R. 1982 Astrophys. J. 252, 553.

Sandage, A. R., Katem, B. and Kristian, J. 1968 Astrophys. J. 153, L129.

Sargent, W. L. W. and Searle, L. 1967 Astrophys. J. 150, L33.

Scalo, J. M. 1986 Fund. Cosmic Phys. 11, 1.

Schwarzschild, M. 1958 Structure and Evolution of the Stars Princeton Univ. Press, Princeton.

Simoda, M. and Iben, I. J. 1970 Astrophys. J. Supp1. 22, 81.

Simoda, M. and Kimura, H. 1968 Astrophys. J. 151, 133. 
Simoda, M. and Tanikawa, K. 1970 Publ. Astron. Soc. Japan 22, 143.

Stetson, P. B. 1979a Astron. J. 84, 1056.

Stetson, P. B. 1979b Astron. J. 84, 1149.

Stryker, L. L. 1984 Astrophys. J. Supp1. 55, 127.

Sweigart, A. V. and Mengel, J. G. 1979 Astrophys. J. 229, 624.

Thomas, H. -C. 1967 Zeit, Astrophys. 67, 420.

Tinsley, B. M. 1980 Fund. Cosmic Phys. 5, 287.

Vandenberg, D. A. 1983 Astrophys. J. Supp1. 51, 29.

Vandenberg, D. A. 1987 in IAU Symposium No. 126, Globular Cluster Systems in Galaxies, J. E. Grindlay and A. G. D. Philip, eds., Reidel, Dordrecht, p. 107.

Yahil, A. and van den Horn, L. 1985 Astrophys. J. 296, 554. 


\section{DISCUSSION}

CAYREL: Do the luminosity functions that you have shown include the horizontal-branch peaks of the evolution?

DEMARQUE: No, the peaks that you saw on the luminosity functions are the Thomas peak.

COHEN: If the observers tell you that $\mathrm{Na}$ and $\mathrm{Al}$ appear to be mixed to the surface from the stellar interior, somewhere between the main sequence and the AGB phase, could you accommodate this within the framework of your models somehow?

DEMARQUE: No, of course not! These $\mathrm{Na}$ and $\mathrm{Al}$ variations must be primordial.

LILLER: The gaps in the sub-giant branch occur close to the point where the onset of chromospheric activity takes place. Have you and Armandroff looked into atmospheric effects for an explanation for the gaps?

DEMARQUE: This is an interesting point. All Armandroff and I did, regarding the surface conditions, was to experiment with different mixing lengths in the convection zone. Indeed, if these is angular momentum hidden in the interior of these stars, this is a phase of evolution where enhanced magnetic activity might be expected since as the convection zone deepens, dynamo activity should be increased.

ROOD: Monte Carlo simulations done by Debe, Zworkin and myself show that reliable determination of the bump location using the differential luminosity function require samples of 1000-2000 stars in the upper 4-1/2 mags. of the RGB. The bump is best identified in the $10 \mathrm{~g}$ of the integrated luminosity function.

DaCOSTA: We have 1200 stars in that range.

ROOD: That's probably enough. Besides the higher metallicity of 47 Tuc makes the bump bigger and at lower luminosity than in most of our simulations. That helps. 